\title{
Attention enhanced capsule network for text classification by encoding syntactic dependency trees with graph convolutional neural network
}

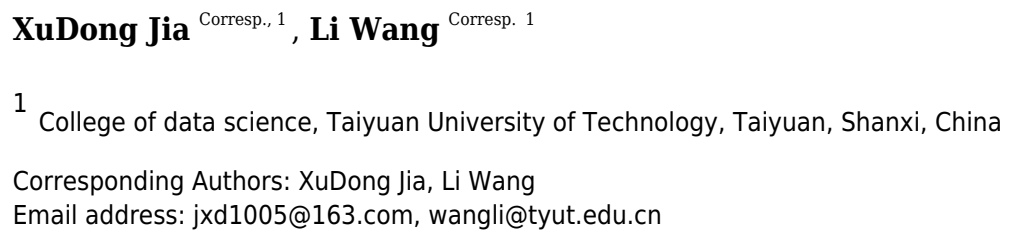

Text classification is a fundamental task in many applications such as topic labeling, sentiment analysis, and spam detection. The text syntactic relationship and word sequence are important and useful for text classification. How to model and incorporate them to improve performance is one key challenge. Inspired by human behavior in understanding text. In this paper, we combine the syntactic relationship, sequence structure, and semantics for text representation, and propose an attention-enhanced capsule network-based text classification model. Specifically, we use graph convolutional neural networks to encode syntactic dependency trees, build multi-head attention to encode dependencies relationship in text sequence, merge with semantic information by capsule network at last. Extensive experiments on five datasets demonstrate that our approach can effectively improve the performance of text classification compared with state-of-the-art methods. The result also shows capsule network, graph convolutional neural network, and multi-headed attention has integration effects on text classification tasks. 


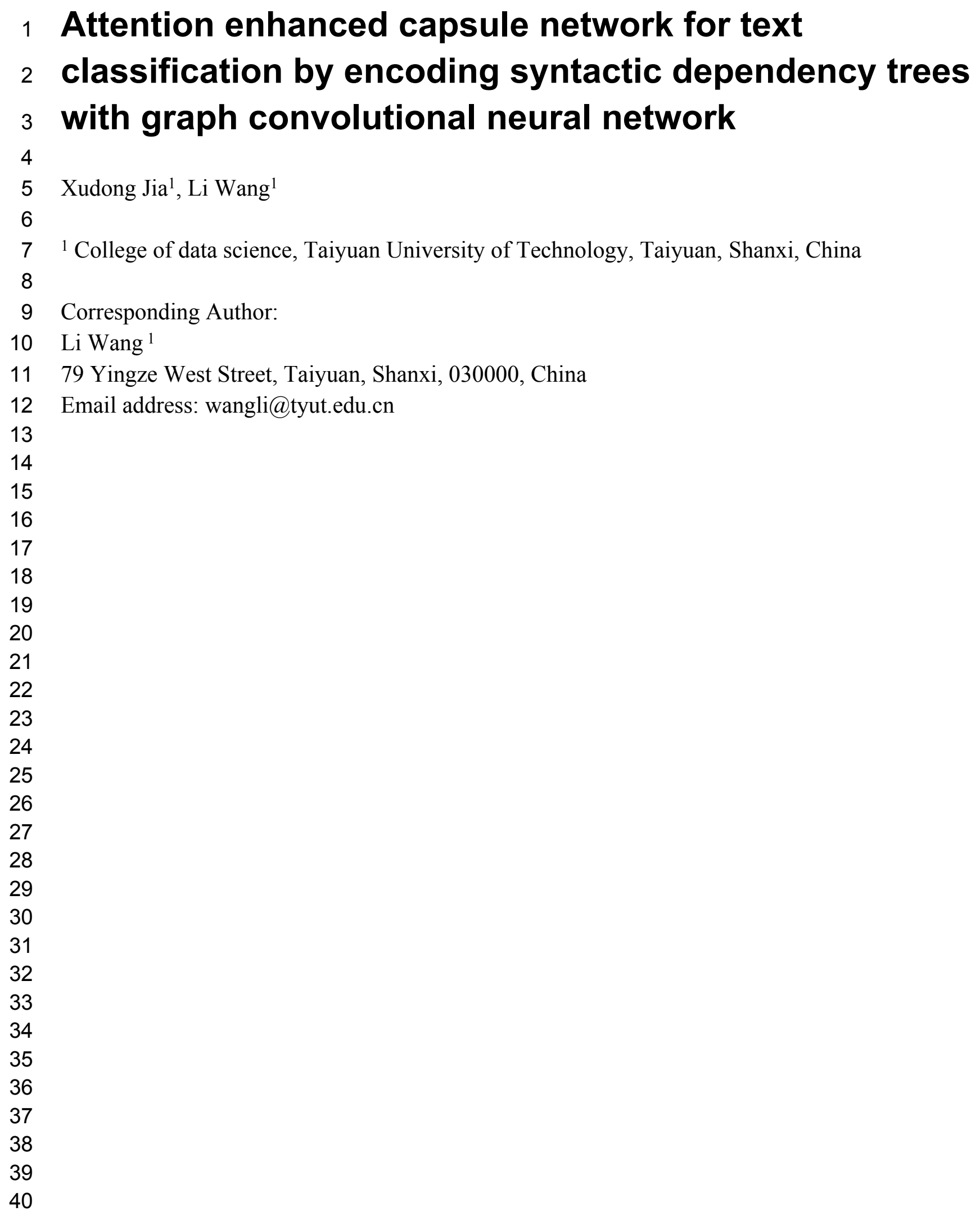

40 
41 Attention enhanced capsule network for text

42

43

44

45

46

47

48

49

50

51

52

53

54

55

56

57

58

59

60

61

62

63

64

65

66

67

68

69

70

71

72

73

74

75

76

77

78

79

\title{
classification by encoding syntactic dependency trees with graph convolutional neural network
}

\begin{abstract}
Text classification is a fundamental task in many applications such as topic labeling, sentiment analysis, and spam detection. The text syntactic relationship and word sequence are important and useful for text classification. How to model and incorporate them to improve performance is one key challenge. Inspired by human behavior in understanding text. In this paper, we combine the syntactic relationship, sequence structure, and semantics for text representation, and propose an attention-enhanced capsule network-based text classification model. Specifically, we use graph convolutional neural networks to encode syntactic dependency trees, build multi-head attention to encode dependencies relationship in text sequence, merge with semantic information by capsule network at last. Extensive experiments on five datasets demonstrate that our approach can effectively improve the performance of text classification compared with state-of-the-art methods. The result also shows capsule network, graph convolutional neural network, and multiheaded attention has integration effects on text classification tasks.
\end{abstract}

\section{Introduction}

Text classification is the basic task of text analysis with broad applications in topic labeling, sentiment analysis, and spam detection. Text representation is one important way for classification. From the analysis of text structure, the text is a sequence of words by certain rules. Sequences of different word orders show different meanings. The sequence structure of a text contains important information about the semantics of the text. Moreover, words in a text are contextual, the distance dependence between words in the sequence structure will also affect the meaning of the text. From the analysis of text composition, the text is composed of words or phrases with different syntactic functions according to certain syntactic relations. For humans, syntactic relations are the basis for editing and reading texts. According to the syntactic relation, we can understand the subject, predicate, and object of the text to promptly understand the semantics of the text. Whether it is text representation or text classification by text representation, it is the key and important question for our research to extract information from the sequence structure and syntactic relations of text.

Traditional methods represent text with handcrafted features, such as bag-of-words (Joachims 1998; Mccallum and Nigam 1998), N-grams (Lin and Hovy 2003), and TF-IDF (Zhang, Yoshida, and Tang 2008), and then adopted machine learning algorithms such as Naive Bayes (Mccallum and Nigam 1998), logistic regression (Genkin and Madigan 2007), support vector machine (Joachims 1998), etc. for classification. These methods ignore the word order and semantic information, which has an important role in understanding the semantics of the text (Pang, Lee, and Vaithyanathan 2002). On the contrary, other methods use word2vec and glove 
80 (Mikolov and Sutskever 2013; Penning- ton, Socher, and Manning 2014) to represent text that 81 can represent the semantic information of words, and then adopted convolutional neural network 82 (CNN) (Kim 2014; Zhang, Zhao, and Lecun 2015; Conneau et al. 2017), long short-term 83 memory networks (LSTM) (Mousa and Schuller 2017), Capsule networks (CapsNet) (Zhao et al. 84 2018) and other deep neural networks for text classification. These methods can effectively 85 encode the word order and semantic information. Kim et al. (Kim 2014) proposed a CNN model 86 that adopted convolution filters to extract local text semantic features for text classification, 87 leading to the model loss of a part of location information (Zhao et al. 2018). Capsule networks 88 with vector neural units and dynamic routing algorithms can effectively overcome the disadvantages of CNN (Sabour, Frosst, and Hinton 2017; Xi, Bing, and Jin 2017). Zhao et al. (Zhao et al. 2018) optimized the capsule networks for text classification (Capsule-A), and further experiments show the effectiveness of capsule networks for text classification. The

92

93

94

95

96

97

98

99

100

101

102

103

104

105

106

107

108

109

110

111

112

113

114

115

116

117

118 characteristics of capsule networks are our starting point. However, this model has limitations in recognizing text with semantic transitions since it cannot encode long-distance dependencies and the global topology, which affects the effectiveness of complex text classification. The attentionbased approach is effective in overcoming the problem of distance dependencies of sequence structure. Both Transformer (Vaswani, Shazeer, and Parmar 2017) and Bidirectional Encoder Representations from Transformers (BERT) (Alaparthi, and Mishra 2020) use multi-head attention as a basic unit to extract text features. It's part of our motivation that it can extract the distance dependencies information of the text from different subspaces.

Syntactic information is a different attribute for text. It describes the syntactic dependency relationship between words in a sentence. It can be represented as the syntactic dependency tree and show the global topology. Figure 1 is a syntactic dependency tree, where 'monkey' is the subject of the predicate 'eats', and 'apple' is its object. GCN is a graph convolutional network that operates on graphs and induces embeddings of nodes based on the properties of their neighborhoods. It can capture the information of the immediate neighbors of nodes at most $\mathrm{K}$ hops away (Marcheggiani and Titov 2017; Duvenaud and Maclaurin 2015). Marcheggiani et al. (Marcheggiani and Titov 2017) used GCN to encode syntactic dependency trees to generate word representations, and combined with long short-term memory networks for semantic role labeling tasks. These works show that GCN can effectively extract syntactic information in syntactic dependency trees. This is another motivation for us.

The text syntactic relationship and word sequence are important and useful for text classification. How to model and incorporate them to improve performance is one key challenge. In this paper, we combine the syntactic relationship, sequence structure, and semantics for text representation, and propose a novel model that utilizes GCN for syntactic relationship, multihead attention for words, and corporate them in capsule network for text classification.

The contributions of this paper can be summarized as follows:

- We incorporate syntactic relationship, sequence structure, and semantics for text representation. 
119

120

121

122

123

124

125

126

127

128

129

130

131

132

133

134

135

136

137

138

139

140

141

142

143

144

145

146

147

148

149

150

151

152

153

154

155

156

157

- We introduce GCN to extract syntactic information for dependencies relationship representation.

- We build multi-head attention to encode the different influences of words to enhance the effect of capsule networks on text classification.

- We show that CapsNet, GCN, and multi-head attention have an integration effect for text classification.

\section{Related work}

\section{Machine learning-based methods}

Early methods adopted the typical features such as bag-of-words (Joachims 1998; Mccallum and Nigam 1998), N-grams (Lin and Hovy 2003), and TF-IDF (Zhang, Yoshida, and Tang 2008) features as input and utilized machine learning algorithms such as support vector achine (SVM) (Joachims 1998), logistic regression (Genkin and Madigan 2007), naive Bayes (NB) (Mccallum and Nigam 1998) for classification. However, these methods ignore the text word order and semantic information, and usually heavily rely on laborious feature engineering.

\section{Deep learning-based methods}

With the introduction of distributed word vector representation (Word Embedding) (Mikolov and Sutskever 2013; Pennington, Socher, and Manning 2014), neural networks-based methods have substantially improved the performance of text classification tasks by encoding text semantics.

CNN was first applied to image processing. Kim et al. (Kim 2014) proposed the CNNbased text classification model (TextCNN). The model uses convolution filters to extract local semantic features and improved upon the state of the art on 4 out of 7 tasks. Zhang X et al. (Zhang, Zhao, and Lecun 2015) proposed the character-level CNN model, which extracts semantic information from character-level original signals for text classification tasks. Conneau et al. (Conneau et al. 2017) proposed very deep convolutional networks to learn the hierarchical representation for text classification. Being a spatially sensitive model, CNN pays a price for the inefficiency of replicating feature detectors on a grid.

Recently, Hinton et al. (Sabour, Frosst, and Hinton 2017) proposed the CapsNet model, which uses vector neural units and dynamic routing update mechanisms, and verified its superiority in image classification. Zhao et al. (Zhao et al. 2018) proposed the text classification model based on CapsNet (Capsule-A), which adopted CapsNet to encode text semantics, and proved that its classification effect is superior to CNN and LSTM. In CapsNet, the feature is represented by a capsule vector instead of a scalar (activation value output by neuron). Different dimensions in a vector can represent different properties of a feature. For a text feature, it often means different meanings in different semantic relations. We use capsules to represent text features to learn the semantic information of different dimensions of text features. On the other hand, the similarity between features at different levels is different. For building a high-level feature, lower levels with high similarity have a higher weight. CapsNet can learn this similarity 
158

159

160

161

162

163

164

165

166

167

168

169

170

171

172

173

174

175

176

177

178

179

180

181

182

183

184

185

186

187

188

189

190

191

192

193

194

195

196

197

relationship through a dynamic routing algorithm. Although CapsNet can effectively improve coding efficiency, it still has limitations in recognizing text with semantic transitions.

Attention mechanisms are widely used in tasks such as machine translation (Vaswani, Shazeer, and Parmar 2017) and speech recognition (Chorowski et al. 2014). Lin et al. (Lin et al. 2017) proposed the self-attention mechanism that can encode long-range dependencies. Vaswani et al. (Vaswani, Shazeer, and Parmar 2017) proposed a machine translation model (Transformer) based on multi-head attention. Alaparthi et al. (Alaparthi, and Mishra 2020) proposed a pretrained model of language representation (BERT) that also takes multi-head attention as its basic component. The basic unit of multi-head attention is scaled dot-product attention. Multi-head attention allows the model to jointly attend to information from different representation subspaces at different positions. Attention is to extract the long-distance dependencies in the text by calculating the similarities between words in the text. The words in the text can express different meanings in different semantic scenarios. The representation of words is different in different semantic spaces. Similar to ensemble learning, multi-head attention can put text in different semantic spaces to calculate attention and get integrated attention. The location information cannot be obtained by relying solely on the attention mechanism, and location information also has an important influence on understanding text semantics. Kim et al. (Kim, Lee, and Jung 2018) proposed a text sentiment classification model combining attention and $\mathrm{CNN}$, but it is still limited by the disadvantages of CNN. Although BERT has been particularly effective on many tasks. But it requires a lot of data and computing resources for pre-training. Therefore, our research is still valuable.

\section{Syntactic information-based Methods}

CNN, RNN, and most deep learning-based methods always utilized word local topology to represent text. Word order and semantic, text syntactic information all have important influences on text classification. Some researchers have done some work on text syntactic information for different tasks. A text is made up of words that represent different syntactic elements, such as subject, predicate, object, and so on. Different syntactic elements are interdependent. A syntax dependency tree is a kind of tree structure, which describes the dependency relationship between words. Figure 1 shows an example of a syntax dependency tree. There are many tools (like StanfordNLP) to generate syntactic dependency trees by analyzing syntactic dependency relations.

Eriguchi et al. (Eriguchi, Tsuruoka, and Cho 2017) adopted RNN to integrate syntactic information for machine translation. Le et al. (Le and Zuidema 2014) introduced RNN to model syntax dependency tree. Miwa et al. (Eriguchi, Tsuruoka, and Cho 2017) used sequential LSTM and tree-LSTM to extract syntactic relations.

The syntactic dependency tree is also a kind of graph data. Bastings et al. (Bastings et al. 2017) used GCN to encode the syntactic dependency tree and combined it with the CNN for machine translation. Marcheggiani et al. (Marcheggiani and Titov 2017) used GCN to encode syntactic dependency trees to generate word representations and combined it with LSTM for the role labeling task. These works show that GCN can effectively extract syntactic information in 
198

199

200

201

202

203

204

205

206

207

208

209

210

211

212

213

214

215

216

217

218

219

220

221

222

223

224

225

226

227

228

229

230

231

232

233

234

235

syntactic dependency trees. The syntactic relationship is presented as a tree structure. A tree is also a form of a graph. Secondly, the sequence structure-based model represents the syntactic tree as a sequence according to some rules of nodes. But the sequence is directional, and nodes in a tree are not sequential. GCN can directly consider the relationship between nodes in a syntax tree.

In summary, we aim to propose a novel model named Syntax-AT-CapsNet that uses multihead attention to extract long-distance dependencies information and that uses GCN to encode syntactic dependency trees to extract syntactic information, which enhances the effect of capsule networks on text classification tasks.

\section{Syntax-AT-CapsNet model}

Our Syntax-AT-CapsNet model consists of the following three modules as depicted in (Fig. 2).

- Attention module. It is composed of an attention layer that adopts multi-head attention. It encodes the dependency relationship between words in the text sequence and important word information to form a text representation.

- Syntax module. It is composed of GCN. It encodes the syntax dependency tree, extracts the syntactic information in the text to for a text representation.

- Capsule network module. It is a capsule network with 5-layer. Based on the text representation output by the Attention module and the Syntax module, it further extracts text semantic and structural information to classify the text.

\section{Input}

The input of the Syntax-AT-CapsNet model is defined as the sentence matrix $\boldsymbol{X}$ :

$$
X=\left[x_{1}, x_{2}, \ldots, x_{L}\right] \in \mathbb{R}^{L \times d},
$$

where $\boldsymbol{x}_{\boldsymbol{i}} \in \mathbb{R}^{d}$ is the word vector of the $i$-th word in the sentence, $L$ is the length of the sentence, and $d$ is the embedding vector size of words.

\section{Attention module}

The Attention module is shown in (Fig. 3). The calculation of attention in this module can be divided into five steps.

First step, linearly transform the input sentence matrix $X$ and divide it into three matrices: $Q$ $\in \mathbb{R}^{L \times d}, \boldsymbol{K} \in \mathbb{R}^{L \times d}, \boldsymbol{V} \in \mathbb{R}^{L \times d}:$

$$
[Q, K, V]=\operatorname{spilt}[X \cdot W],
$$

where $\boldsymbol{W} \in \mathbb{R}^{d \times 3 d}$ is the transform matrix, and spilt denotes the division operation.

Second step, linearly projects the matrices $\boldsymbol{Q}, \boldsymbol{K}, \boldsymbol{V}$ onto $h$ different linear subspaces: 
236 where $\boldsymbol{Q}_{\boldsymbol{i}} \in \mathbb{R}^{L \times \frac{d}{h}}, \boldsymbol{K}_{\boldsymbol{i}} \in \mathbb{R}^{\mathrm{L} \times \frac{\mathrm{d}}{h}}, \boldsymbol{V}_{\boldsymbol{i}} \in \mathbb{R}^{L \times \frac{d}{h}}$ is the mapping of $\boldsymbol{Q}, \boldsymbol{K}, \boldsymbol{V}$ on the $i$-th subspace, $\boldsymbol{W}^{\boldsymbol{Q}_{\boldsymbol{i}}} \in$ 237 $\mathbb{R}^{d \times \frac{d}{h}}, \boldsymbol{W}^{\boldsymbol{K}_{\boldsymbol{i}}} \in \mathbb{R}^{d \times \frac{d}{h}}, \boldsymbol{W}^{\boldsymbol{V}_{\boldsymbol{i}}} \in \mathbb{R}^{d \times \frac{d}{\bar{h}}}$ is the transform matrix, $i=[1, \ldots, h]$. The purpose of this step is 238 to compute multiple attention values in parallel. At the same time, the dimension of input matrix 239 is reduced to reduce the calculation pressure caused by multiple calculation.

240 Third step, calculate the attention on each subspace in parallel:

where $\boldsymbol{h e a d}_{\boldsymbol{i}}$ is the attention value on the $i$-th subspace, softmax denotes the softmax function (Vaswani, Shazeer, and Parmar 2017). In fact, $\boldsymbol{Q}_{\boldsymbol{i}}$ and $\boldsymbol{K}_{\boldsymbol{i}}$ represents the sentence matrix on the subspace. It's divided by $\sqrt{d}$ in case the dot product gets too big. The weight of the sentence matrix on the subspace $\mathbf{V}_{\boldsymbol{i}}$ is obtained by calculating the dot product of $\boldsymbol{Q}_{\boldsymbol{i}}$ and $\boldsymbol{K}_{\boldsymbol{i}}$ and using a softmax function.

Fourth step, concat the attention values on each subspace and get the attention value of the entire sentence through linear transformation:

$$
\text { Multi }_{\text {head }}=\text { concat }\left(\text { head }_{1}, \ldots, \text { head }_{h}\right) W^{M},
$$

where $\boldsymbol{W}^{\boldsymbol{M}} \in \mathbb{R}^{d \times d}$ is the transform matrix, Multi_head is the attention value of the entire sentence, and concat denotes the concat operation.

The final step, Connect the attention value $\mathbf{M u l t i} \_$head to the original sentence matrix $\boldsymbol{X}$ to get the sentence matrix output by the module:

$$
X_{1}=\text { residual_Connect }\left(X, M u l t i \_ \text {head }\right),
$$

where $\boldsymbol{X}_{\mathbf{1}} \in \mathbb{R}^{L \times d}$ is the output of the attention module, and residual_Connect denotes the residual connection operation.

\section{Syntax module}

The Syntax module is shown in (Fig. 4). The Syntax module uses GCN to encode syntactic dependency trees, which can encode syntactic relationships between words in a text into word vectors. The module first needs to use a natural language processing tool (we adopted StanfordNLP) to generate the syntactic dependency tree of the input sentence, and construct its adjacency matrix. The adjacency matrix construction algorithm in this paper is shown in Algorithm 1. Since the syntactic relationship between word nodes in the syntactic dependency tree has direction, when constructing the adjacency matrix, the syntactic dependency tree is used as a directed graph. In addition, in order not to be disturbed by its word vector, the node is not provided with a self-loop. As shown in (Fig. 5), the adjacency matrix corresponds to the example sentence "The Monkey eats an apple" shown in (Fig. 1) and is generated by our method. The input sentence matrix and adjacency matrix are further passed through the GCN to obtain a text representation containing syntactic information. 


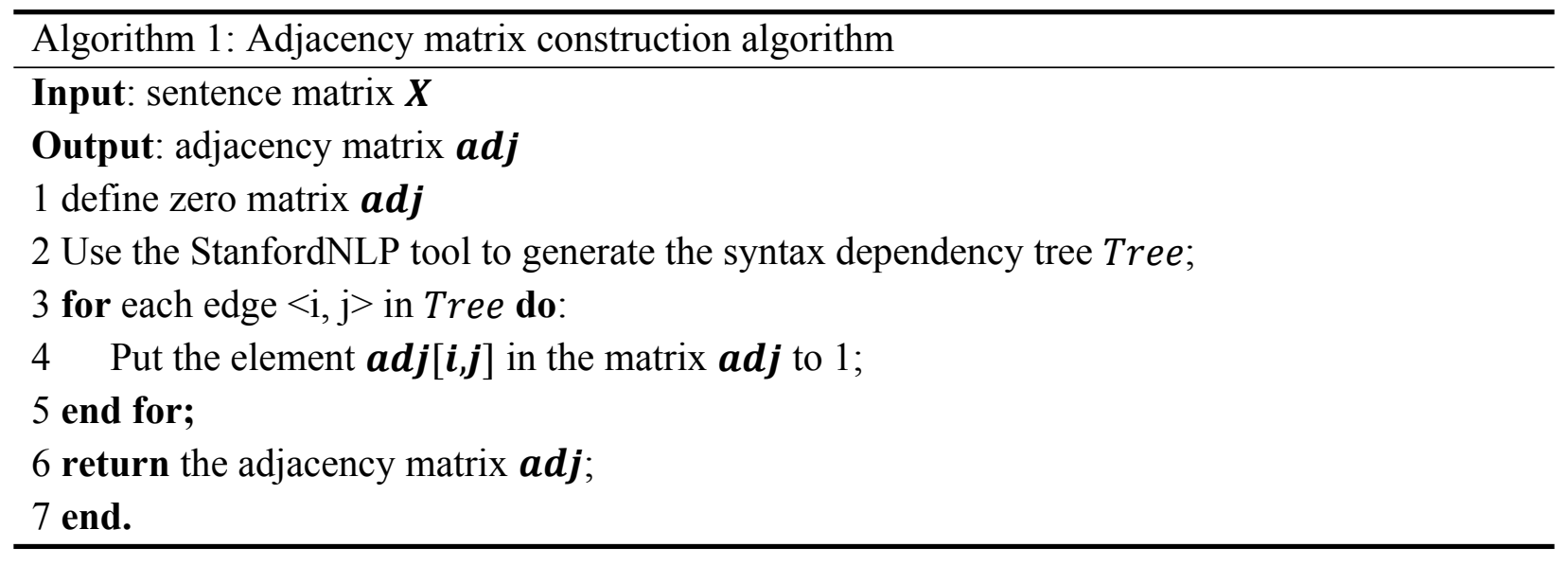

275

276

277

278

279

280

281

282

283

284

285

286

287

288

289

290

291

292

293

294

295

296

297

298

299

300

301

The calculation in the Syntax module can be divided into two steps.

First step, use StanfordNLP tool to generate syntactic dependency tree and construct adjacency matrix $\boldsymbol{a d j}$.

Second step, perform a two-layer graph convolution operation on the input sentence matrix $X$ and adjacency matrix $\boldsymbol{a d j}$ :

$$
\begin{gathered}
G=\operatorname{relu}\left((X \cdot \operatorname{adj}) W^{t 1}\right), \\
X_{2}=\operatorname{softmax}\left((G \cdot a d j) W^{t 2}\right),
\end{gathered}
$$

where $\boldsymbol{G} \in \mathbb{R}^{L \times d}$ is the output of the first layer graph convolution operation, $\boldsymbol{X}_{\mathbf{2}} \in \mathbb{R}^{L \times d}$ is the output of the second layer graph convolution operation, and $\boldsymbol{a d j} \in \mathbb{R}^{L \times L}$ is adjacent matrix, $W^{t 1}$ 、 $W^{t 2} \in \mathbb{R}^{d \times d}$ are parameter matrices. In operation (7), the adjacency matrix and sentence matrix go through the first level graph-convolution operation. The relationship between nodes directly connected is obtained. Then, through operation (8), the relationship between nodes indirectly connected nodes is calculated.

\section{Capsule network module}

The capsule network module is composed of a fusion layer, a convolution layer, a primary capsule layer, a convolution capsule layer, and a fully connected capsule layer. It uses the text representation output by the attention module and the syntax module as input to further extract features. Each layer in the module can extract different levels of features. By further combining low-level features to obtain higher-level features, and finally form a feature representation of the entire text for classification.

The first layer is the fusion layer, put the text representations $\boldsymbol{X}_{\mathbf{1}}$ and $\boldsymbol{X}_{\mathbf{2}}$ output by the syntax module and the attention module into a single layer network:

$$
X_{3}=W^{f 1} \cdot X_{1}+W^{f 2} X_{2}
$$

where $W^{f 1}, W^{f 2} \in \mathbb{R}^{L}$. Two sentence matrices are combined by linear transformation through this step. 
302

303

304

305

306

307

308

309

310

311

312

313

314

315

316 317 is obtained by operation (13):

318

319

320

321

322

323

324

325

326

327

328

329

330

331

332

333 obtain a prediction vector:

The second layer is the convolutional layer, which extracts N-gram phrase features at different positions in the text. This layer uses $k_{1}$ convolution filters to perform convolution on the sentence matrix $\boldsymbol{X}_{\mathbf{3}}$ to obtain the N-gram feature matrix $\boldsymbol{M}$ :

$$
M=\left[M_{1}, M_{2}, \ldots, M_{k_{1}}\right] \in \mathbb{R}^{(L-N+1) \times k_{1}}
$$

where $\boldsymbol{M}_{\boldsymbol{k}_{\mathbf{1}}}=\left[m_{1}, m_{2}, \ldots, m_{L-N+1}\right] \in \mathbb{R}^{L-N+1}$ is the $k_{1}$-th column vector in $\boldsymbol{M}$, each element $m_{i}$ in this vector is obtained by operation (11):

$$
m_{i}=f\left(W^{c 1} \cdot x_{i: i+N-1}+b_{1}\right),
$$

where $f$ denotes the nonlinear activation function, $W^{c 1} \in \mathbb{R}^{N \times d}$ is the $k_{1}$-th convolution filter, $\boldsymbol{x}_{\boldsymbol{i}: \boldsymbol{i}+\boldsymbol{N}-\mathbf{1}}$ denotes that $N$-word vectors in the sentence are connected in series, $b_{1}$ is bias item. The original features are extracted by convolution in this step.

The third layer is the primary capsule layer, which combines the N-gram phrase features extracted at the same location as capsules. This layer uses $k_{2}$ transformation matrices to transform the feature matrix $M$ into the primary capsule matrix $P$ :

$$
\boldsymbol{P}=\left[\boldsymbol{P}_{1}, \boldsymbol{P}_{2}, \ldots, \boldsymbol{P}_{k_{2}}\right] \in \mathbb{R}^{(L-N+\mathbf{1}) \times \boldsymbol{k}_{2} \times \boldsymbol{l}},
$$

where $\boldsymbol{P}_{\boldsymbol{k}_{\mathbf{2}}}=\left[\boldsymbol{p}_{\mathbf{1}}, \boldsymbol{p}_{2}, \ldots, \boldsymbol{p}_{\boldsymbol{L}-\boldsymbol{N}+\mathbf{1}}\right] \in \mathbb{R}^{(L-N+1) \times l}$ is the $k_{2}$-th column capsule in $P$, each capsule

$$
p_{i}=g\left(W^{c 2} \cdot M^{i}+b_{2}\right),
$$

where $g$ denotes the nonlinear compression function, $W^{c 2} \in \mathbb{R}^{k_{2} \times 1 \times l}$ is the $k_{1}$ th transformation matrix, $\boldsymbol{M}^{\boldsymbol{i}}$ is the $i$-th row vector in $\boldsymbol{M}$, and $b_{2}$ is bias item. The primary capsules are constructed by linearly transforming the original features of the same location.

The fourth layer is the convolution capsule layer, which uses a shared transformation matrix to extract local capsules, similar to the convolution layer. This layer uses $k_{3}$ transformation matrices to perform capsule convolution operation on $P$ to obtain the capsule matrix $U$ :

$$
U=\left[U_{1}, U_{2}, \ldots, U_{k_{3}}\right] \in \mathbb{R}^{\left(L-N-N_{1}+2\right) \times k_{3} \times l},
$$

where $\boldsymbol{U}_{\boldsymbol{k}_{\mathbf{3}}}=\left[\boldsymbol{u}_{\mathbf{1}}, \boldsymbol{u}_{\mathbf{2}}, \ldots, \boldsymbol{u}_{\boldsymbol{L}-\boldsymbol{N}-\boldsymbol{N}_{\mathbf{1}}+\mathbf{2}}\right] \in \mathbb{R}^{\left(L-n_{2}+1\right) \times l}$ is the $k_{3}$-th column capsule in $U$, each capsule is obtained from the $N_{1}$ line capsules in P:

$$
\mathbf{u}_{r} \leftarrow P^{i: i+\left(N_{1} \times k_{2}\right)-1}
$$

where $\boldsymbol{P}^{i: i+\left(N_{1} \times k_{2}\right)-1}$ represents $N_{1}$ rows of capsules, and each capsule is linearly converted to

$$
\hat{p}_{i}=W^{c 3} \cdot p_{i}+\hat{b}_{i}
$$

where $W^{c 3} \in \mathbb{R}^{l \times l}$ is the $k_{3}$-th conversion matrix, $\hat{b}_{i}$ is the offset term. It's the same thing as convolution, except the basic units become capsules. And the prediction vector is operated (17): 
334

335

336

337

338

339

340

341

342

343

344

345

346

347

348

\section{9}

350

351

352

353

354

$$
\mathbf{u}_{r}=\boldsymbol{g}\left(\boldsymbol{\Sigma}_{i} \mathbf{c}_{i} \cdot \hat{\boldsymbol{p}}_{\boldsymbol{i}}\right),
$$

where $g$ is a nonlinear activation function, $c_{i}$ is the coupling coefficient, which is updated with a dynamic routing algorithm (Zhao et al. 2018). The similarity between the primary capsules and the generated convolutional capsules is different within a window. A primary capsule with a high similarity should be given a higher weight. Equation (17) is based on this principle.

The last layer is the fully connected capsule layer, which is used to form the capsule $\mathrm{Y}$ representing the category:

$$
Y=\left[y_{1}, y_{2}, \ldots, y_{j}\right] \in \mathbb{R}^{j \times l},
$$

where $\boldsymbol{y}_{\boldsymbol{j}} \in \mathbb{R}^{l}$ denotes the capsule of the $j$-th category. The capsules in $U$ are linearly transformed (16) to obtain the prediction vector $\boldsymbol{u}_{j \mid r}$, and the operation (17) is performed to obtain $\boldsymbol{y}_{\boldsymbol{j}}$. The fully connected capsule layer is shown in (Fig. 6), it can also represent a convolution window operation at the fourth layer.

Finally, the modulus of the capsule vector representing the category in the fully connected capsule layer is taken as the probability of belonging to the category.

\section{Syntax-AT-CapsNet learning algorithm}

The learning algorithm of Syntax-AT-CapsNet is shown in Algorithm 2. When the model is learning, the coupling coefficients are updated by the dynamic routing algorithm, and the global parameters of the model are updated by the back propagation algorithm. Then the trained Syntax-AT-CapsNet model parameters can be obtained. During prediction, the classification results can be obtained through the sequential calculation of each module in the model.

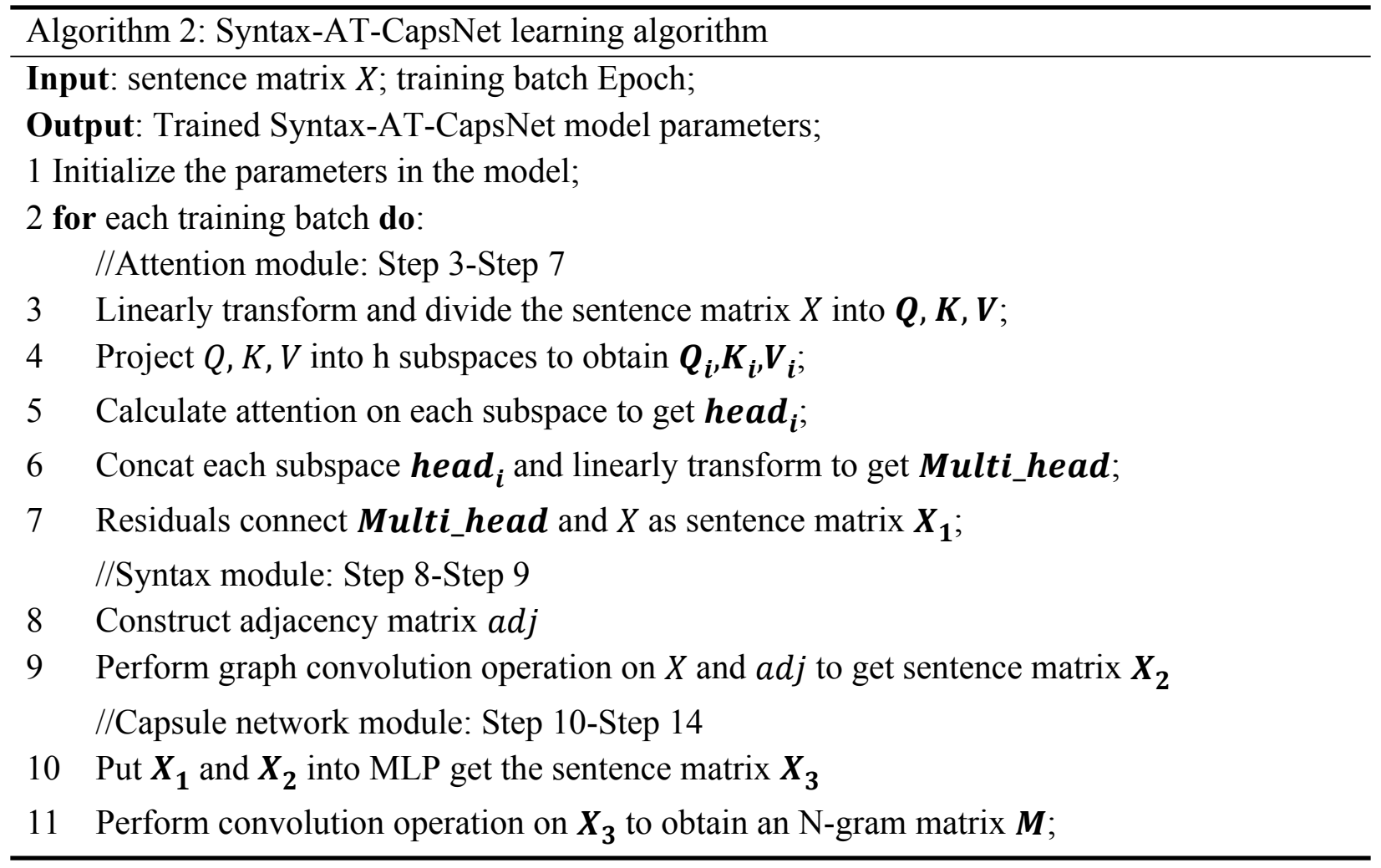


12 Convert the N-gram feature matrix $\boldsymbol{M}$ to the capsule matrix $\boldsymbol{P}$;

13 Perform capsule convolution and routing on $\boldsymbol{P}$ to obtain the capsule matrix $\boldsymbol{U}$;

14 Perform capsule calculation and routing on $\boldsymbol{U}$ to obtain category capsule $\boldsymbol{Y}$;

15 Calculate loss and update parameters by back propagation;

16 end for;

17 return the trained Syntax-AT-CapsNet model parameters;

18 end.

355

356

357

358

359

360

361

362

363

364

365

366

367

368

369

370

371

372

373

374

375

376

377

378

379

380

381

382

383

384

385

386

\section{Experimental details}

We did extensive experiments to verify the effect of Syntax-AT-CapsNet model on the single label and multi-label text classification tasks and designed more ablation experiments to demonstrate the role of each module.

\section{Data sets}

We choose the following five datasets in our experiments: movie reviews (MR) (Miwa and Bansal 2016), subjectivity dataset (Subj) (Pang and Lee 2004), customer review (CR) (Hu and Liu 2004), Reuters-21578 (Lewis 1992).

The data in MR, Subj, and CR have two categories and are used for the single-label classification tasks, where MR and Subj are composed of movie sentiment review data, CR is composed of product reviews from Amazon and Cnet. The Reuters-21578 test set consists of Reuters news documents. We selected 10788 news documents under the 8 category labels related to economic and financial topics in Reuters-21578 and further divided them into two subdatasets (Reuters-Full and Reuters-Multi). In Reuters-Full, all texts are kept as the test set, and in Reuters-Multi, only multi-label texts are kept as the test set. The experimental data description is shown in Table 1.

\section{Evaluation index}

Exact Match Ratio (ER), Micro Averaged Precision (Precision), Micro Averaged Recall (Recall), and Micro Averaged F1 (F1) were used as evaluation indexes in the experiment. Accuracy is used instead of ER in the single-label classification.

\section{Parameter setting}

The experimental parameters of our work are as follows. In the model, input a 300-dimensional word2 vec word vector $(d=300)$. The attention module uses two heads of attention $(h=2)$. The first layer of the capsule network module uses 32 convolution filters $\left(k_{1}=32\right)$, the window size is $3(N=3)$. The second layer uses 32 transformation matrices $\left(k_{2}=32\right)$ and 16-dimensional capsule vectors $(l=16)$. The third layer uses 16 conversion matrices $\left(k_{3}=16\right)$, the window size is $3\left(N_{1}=3\right)$. The last layer uses 9 capsule vectors $(j=9)$ to represent 9 classes. 
393

394

395

396

397

398

399

400

401

402

403

404

405

406

407

408

409

410

411

412

413

414

415

416

417

418

419

420

421

422

423

424

425

In model training, mini-batch with a size of 25 (batch_size) are used, the training batch is controlled to 20 (Epoch $=20)$, and the learning rate is set to 0.001 (learning_rate $=0.001$ ). During the model test, for the single label classification task, the category label corresponding to the capsule vector with the largest module length is taken. For the multi-label classification tasks, the category labels corresponding to capsule vectors with a modulus length greater than 0.5 are taken.

\section{Benchmark model}

In this paper, TextCNN, Capsule-A, and AT-CapsNet are used as benchmark models for comparative experiments. TextCNN is a classic model of text classification based on CNN, which is representative. Capsule-A is a text classification model based on capsule networks. ATCapsNet is a multi-headed attention capsule network text classification model.

\section{Experimental results and discussion}

\section{Performance on single-classification and multi-classification tasks}

The experimental result of single-label classification is shown in Table 2, and the multi-label classification experiment result is shown in Table 3.

It can be observed from the experimental results:

- Compared with the benchmark model (Table 2), our model achieved the best results of accuracy, Recall, and F1 on the three binary classification data sets ER, Subj, CR. Compared with AT-CapsNet (a multi-headed attention capsule network that does not introduce syntax), it is found that the three data sets have a significant improvement, which proves the value of introducing syntactic information in this article.

- Compared with the benchmark models (Table 3), on the two multi-label data sets ReutersFull and Reuters-Multi, our model has achieved competitive results in four evaluation indicators. And achieves the best results in ER, Recall, and F1, which indicates the effectiveness of the model in the classification of multi-tag texts.

That is, our model has better effects on multi-label and single-label classification tasks than benchmark methods. As described in the introduction, the problems with the baseline model are the key to our research. This shows that our model overcomes the shortcomings of these models to a certain extent. That's the purpose of our work.

\section{Syntax module verification experiment}

To show the effect of the syntax module, we did the following experiments and the experimental results are shown in Table 4.

We can see that when the syntactic module is added to the benchmark model, the four evaluation indicators have been significantly improved, which shows that the syntactic module of this article can effectively improve the effect of text classification tasks. It also proves the 
426 feasibility and value of extracting syntactic information with graph convolutional neural

427

428

429

430

431

432

433

434

435

436

437

438

439

440

441

442

443

444

445

446

447

448

449

450

451

452

453

454

455

456

457

458

459

460

461

462

463

464

465

networks. This also shows that we are right to learn from human reading behavior.

\section{Module ablation experiment}

The results of the module ablation experiment are shown in Table 5 and Table 6.

From the above experimental results, we can draw the following conclusions:

- When controlling a single module (Table 5), the ablation of each module will cause the classification effect to decrease to varying degrees, which shows that each module in our model has a certain role in improving the text classification effect. In addition, by comparing the reduced values, it was found that the capsule network had the greatest influence, followed by attention, and the syntax module was smaller, which indicated the correctness and value of taking the capsule network as the core module in this paper.

- When two modules are controlled (Table 6), the ablation of any two modules will cause the classification effect to decrease to varying degrees. Among them, the ablation syntax and capsule network modules have the greatest influence, and the ablation syntax and attention module have the second influence, and the ablation attention and the capsule network module has the least influence, which shows that the syntactic module can function to the greatest extent when combined with other modules. It also shows that the motivation of using graph neural networks to encode syntactic information and other models is correct.

- It can be seen from the above that when the attention module, the syntax module, or the capsule network module in the model of this article is removed or partially removed, the effectiveness of the model has declined to vary degrees. Since the syntactic module uses graph convolutional neural networks, the above experiments also prove that graph convolutional neural networks, capsule networks, and multi-head attention have an integrated effect on text classification tasks. This also shows that we are on the right track in building the model.

\section{Conclusions}

This paper proposes an enhanced capsule network text classification model Syntax-AT-CapsNet for text classification tasks. The model first uses graph convolutional neural networks as submodules to encode syntactic dependency trees, extract syntactic information in text, and further integrate with sequence information and dependency relationships, thereby improving the effect of text classification. Through model classification effect verification experiment, syntax module verification experiment, and module ablation experiment, the effect of the model in this paper on text classification and multi-label text classification task is verified, the function of syntax module is demonstrated, and the integrated effect of graph convolutional neural network, capsule network, and multi-head attention is proved. Future work will further optimize the model for other downstream tasks of text classification.

\section{References}

Joachims, T. 1998. Text categorization with Support Vector Machines: Learning with many relevant features. In European Conference on Machine Learning, 137-142. 
466 Mccallum, A.; and Nigam, K. 1998. A comparison of event models for naive bayes text 467 classification. In AAAI-98 workshop on learning for text categorization, 137-142.

468 Lin, C-Y.; and Hovy, E. 2003. Automatic Evaluation of Summaries Using N-gram Co-

469 occurrence Statistics. In Proceedings of the 2003 Conference of the North American Chapter of

470 the Association for Computational Linguistics on Human Language Technology, 291-304.

471 Zhang, W.; Yoshida, T.; and Tang, X. 2008. TFIDF, LSI and multi-word in information retrieval

472 and text categorization. In IEEE International Conference on Systems, 108-113.

473 Genkin, A.; and Madigan, L. D. 2007. Large-Scale Bayesian Logistic Regression for Text

474 Categorization. 49(3): 1019-1026.

475 Pang, B.; Lee, L.; and Vaithyanathan, S. 2002. Thumbs up? Sentiment Classification using

476 Machine Learning Techniques. In Con Empirical Methods in Natural Language Processing, 79-

47786.

478 Mikolov, T.; and Sutskever, K. e. a., I. Chen. 2013. Distributed Representations of Words and

479 Phrases and their Compositionality. 26: 3111-3119.

480 Pennington, J.; Socher, R.; and Manning, C. D. 2014. Glove: Global Vectors for Word

481 Representation. In Conferenceonference on Empirical Methods in Natural Language Processing, 482 1532-1543.

483 Kim, Y. 2014. Convolutional neural networks for sentence classification. arXiv:1408.5882.

484 Zhang, X.; Zhao, J.; and Lecun, Y. 2015. Character-level Convolutional Networks for Text

485 Classification. In Neural Information Processing Systems.

486 Conneau, A.; Schwenk, H.; Barrault, L.; and et al. 2017. Very Deep Convolutional Networks for

487 Text Classification. In European Chapter of the Association for Computational Linguistics,

488 1107-1116.

489 Mousa, A.; and Schuller, B. 2017. Contextual Bidirectional Long Short-Term Memory Recurrent

490 Neural Network Language Models: A Generative Approach to Sentiment Analysis. In European

491 Chapter of the Association for Computational Linguistics, 1023-1032.

492 Zhao, W.; Ye, J.; Yang, M.; and et al. 2018. Investigating Capsule Networks with Dynamic

493 Routing for Text Classification. In Conference on Empirical Methods in Natural Language

494 Processing, 3110-3119.

495 Sabour, S.; Frosst, N.; and Hinton, G. E. 2017. Dynamic Routing Between Capsules.

496 Xi, E.; Bing, S.; and Jin, Y. 2017. Capsule Network Performance on Complex Data.

497 arXiv:1712.03480.

498 Vaswani, A.; Shazeer, N.; and Parmar, N. e. a. 2017. Attention is All you Need.

499 Alaparthi, S.; Mishra, M. 2020. Bidirectional Encoder Representations from Transformers

500 (BERT): A sentiment analysis odyssey. arXiv: 2007.01127

501 Marcheggiani, D.; and Titov, I. 2017. Encoding sentences with graph convolutional networks for 502 semantic role labeling. In The 2017 Conference on Empirical Methods in Natural Language

503 Processing, 1506-1515. 
504 Duvenaud, D. K.; and Maclaurin, J. e. a., D. Iparraguirre. 2015. Convolutional networks on 505 graphs for learning molecular fingerprints. In Neural Information Processing Systems, 22245062232.

507 Bastings, J.; Titov, I.; Aziz, W.; and et al. 2017. Graph Convolutional Encoders for Syntax-aware

508 Neural Machine Translation. In Proceedings of the 2017 Conference on Empirical Methods in 509 Natural Language Processing.

510 Chorowski, J.; Bahdanau, D.; Cho, K.; and et al. 2014. End-to-end Continuous Speech

511 Recognition using Attention-based Recurrent NN: First Results. arXiv:1412.1602.

512 Lin, Z.; Feng, M.; Santos, C. N. D.; and et al. 2017. A Structured Self-attentive Sentence

513 Embedding. In International Conference on Learning Representations.

514 Kim, Y.; Lee, H.; and Jung, K. 2018. AttnConvnet at SemEval-2018 Task 1: Attention-based

515 Convolutional Neural Networks for Multi-label Emotion Classification. In International

516 Workshop on Semantic Evaluation.

517 Eriguchi, A.; Tsuruoka, Y.; and Cho, K. 2017. Learning to parse and translate improves neural

518 machine translation. In the 55th Annual Meeting of the Association for Computational

519 Linguistics, 72-78.

520 Le, P.; and Zuidema, W. 2014. The Inside-Outside Recursive Neural Network model for

521 Dependency Parsing. In the 2014 Conference on Empirical Methods in Natural Language

522 Processing, 729-739.

523 Eriguchi, A.; Tsuruoka, Y.; and Cho, K. 2017. Learning to parse and translate improves neural

524 machine translation. In the 55th Annual Meeting of the Association for Computational

525 Linguistics, 72-78.

526 Miwa, M.; and Bansal, M. 2016. End-to-end relation extraction using 1stms on sequences and 527 tree structures. In the 54th Annual Meeting of the Association for Computational Linguistics, $528 \quad 1105-1116$.

529 Pang, B.; and Lee, L. 2004. A sentimental education: Sentiment analysis using subjectivity. In 530 the 54th Annual Meeting of the Association for Computational Linguistics, 271-278.

$531 \mathrm{Hu}, \mathrm{M}$; ; and Liu, B. 2004. Mining and summarizing customer reviews. In Tenth Acm Sigkdd

532 International Conference on Knowledge Discovery and Data Mining.

533 Lewis, D. D. 1992. An Evaluation of Phrasal and Clustered Representations on a Text

534 Categorization Task. In Proceedings of the 15th Annual International ACM SIGIR Conference 535 on Research and Development in Information Retrieva. 
Figure 1

The example of Syntactic dependency tree, where 'monkey' is the subject of the predicate 'eats', and 'apple' is its object. 


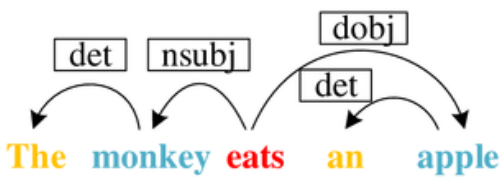


Figure 2

The architecture of the Syntax-AT-CapsNet model, which consists of three modules, Attention module,Syntax module and Capsule network module. 


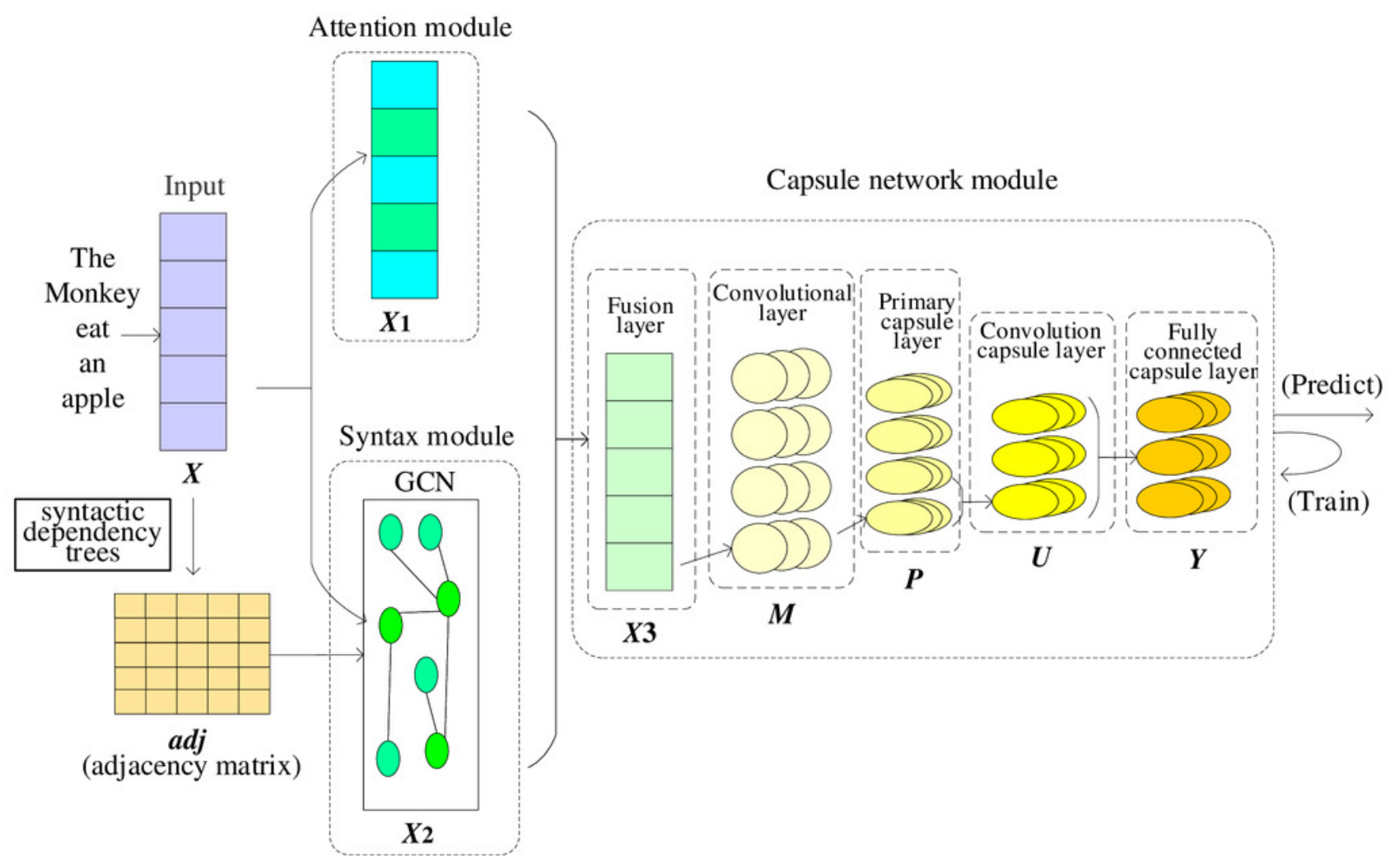




\section{Figure 3}

The architecture of the Attention module, which consists of three parallel attention computing units. 


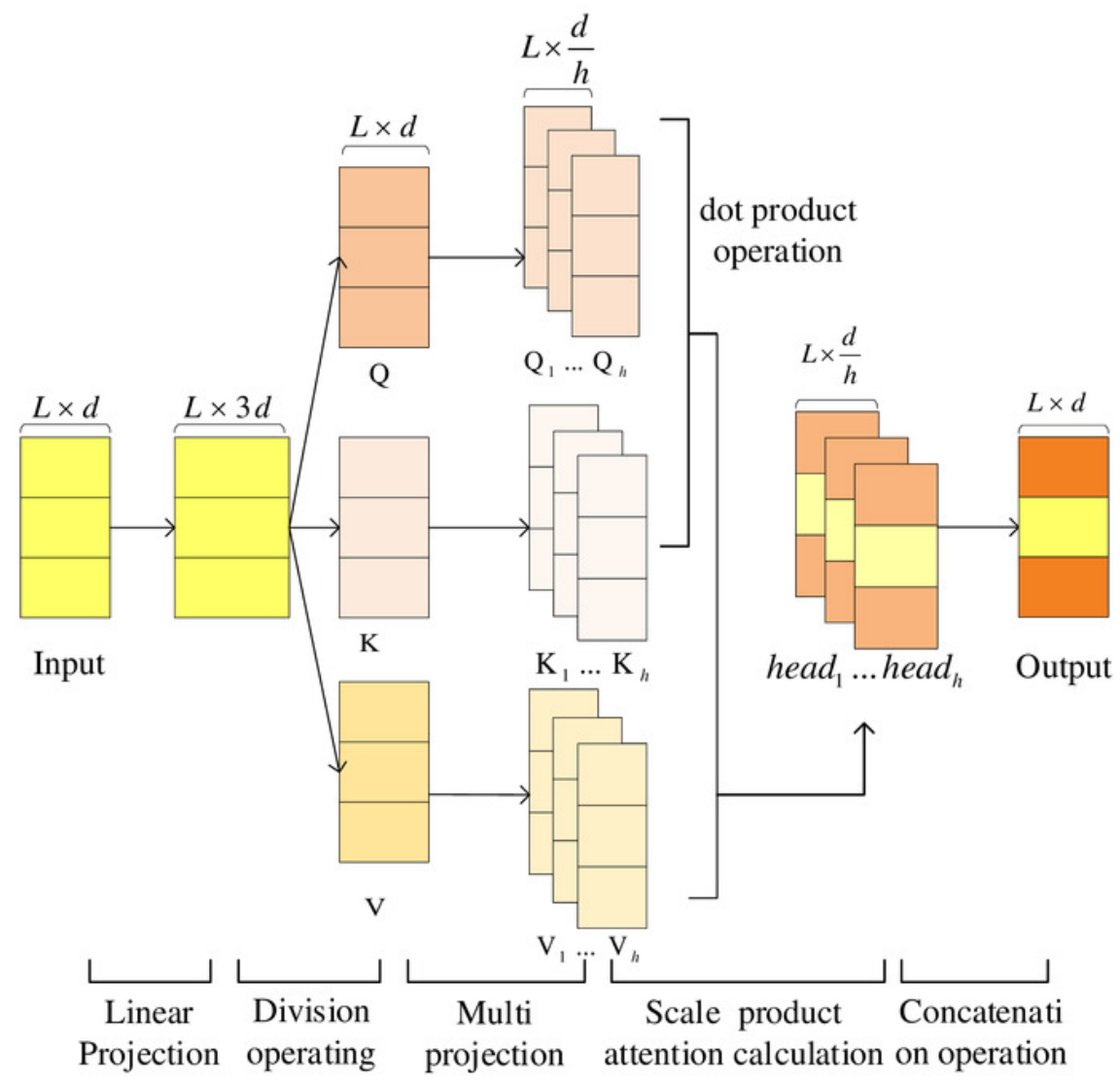


Figure 4

The architecture of the Syntax module, which includes the construction of the syntactic dependency tree and the GCN unit. 


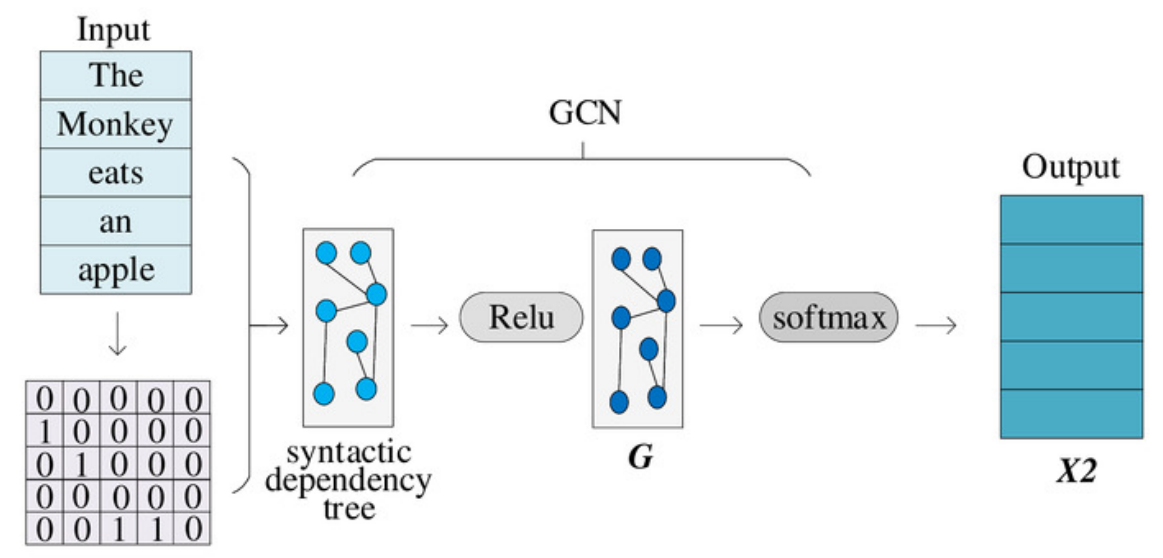

Adjacency matrix 
Figure 5

The example of adjacency matrix for a syntactic dependent tree. 


\begin{tabular}{|c|c|c|c|c|c|}
\hline \multirow[b]{2}{*}{ The } & \multicolumn{5}{|c|}{ The Monkey eat an appl } \\
\hline & 0 & 0 & 0 & 0 & 0 \\
\hline Monkey & 1 & 0 & 0 & 0 & 0 \\
\hline eat & 0 & 1 & 0 & 0 & 0 \\
\hline an & 0 & 0 & 0 & 0 & 0 \\
\hline apple & 0 & 0 & 1 & 1 & 0 \\
\hline
\end{tabular}




\section{Figure 6}

The architecture of fully connected capsule layer, its input is the output of the previous capsule layer 


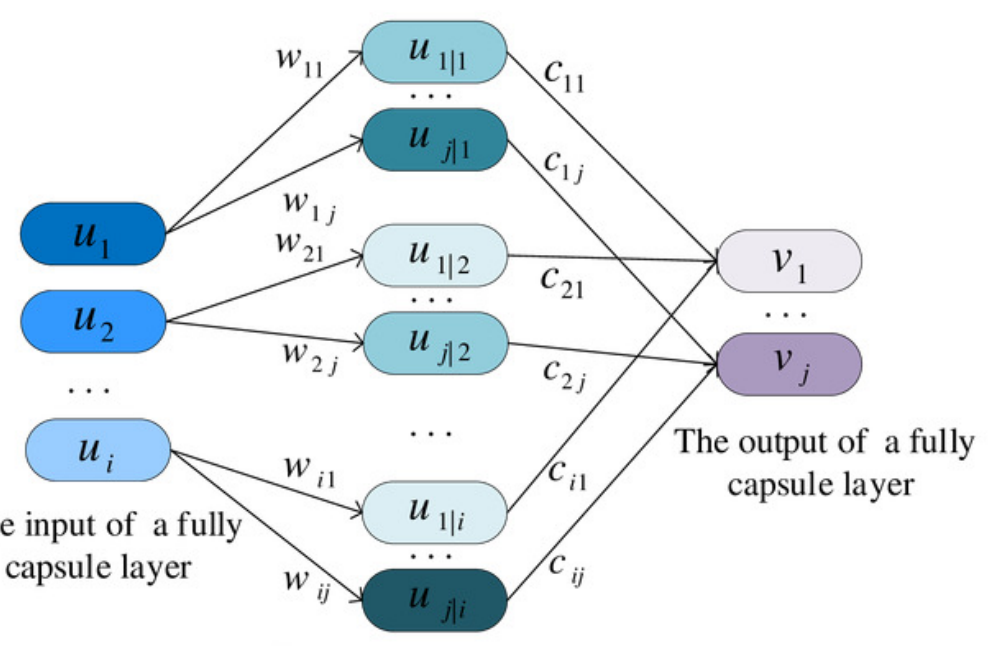

The prediction of a fully

capsule layer 


\section{Table $\mathbf{1}$ (on next page)}

Data description, it describes the number of training sets, validation sets and test sets of five kinds of data sets, the number of categories of each data set, and whether the data set belongs to single-label data or multi-label data. 


\begin{tabular}{cccc}
\hline Data set & Train / Vec / Test & Num of category & Test label \\
\hline MR & $8632 / 960 / 1066$ & 2 & Single \\
CR & $3053 / 340 / 377$ & 2 & Single \\
Subj & $8099 / 900 / 1000$ & 2 & Multi \\
Reuters-Full & $5000 / 500 / 2500$ & 8 & Single / Multi \\
Reuters-Multi & $5000 / 500 / 300$ & 8 & Multi \\
\hline
\end{tabular}

1 


\section{Table 2 (on next page)}

The experimental result of single label classification. Our model is compared with the benchmark model on three single-label datasets. The evaluation index is accuracy. 


\begin{tabular}{ccccc}
\hline Evaluation index & Accuracy(\%) & Precision(\%) & Recall(\%) & F1(\%) \\
\hline Data set & \multicolumn{5}{c}{ MR } \\
\hline TextCNN & 73.0 & $\mathbf{9 2 . 3}$ & 87.6 & 89.9 \\
Capsule-A & 74.0 & 90.2 & 89.8 & 90.0 \\
AT-CapsNet & 74.8 & 91.5 & 90.0 & 90.7 \\
Syntax-AT-Capsule(ours) & $\mathbf{7 5 . 3}$ & 92.0 & $\mathbf{9 0 . 8}$ & $\mathbf{9 1 . 4}$ \\
\hline Data set & \multicolumn{5}{c}{ Subj } \\
\hline TextCNN & 81.6 & $\mathbf{9 6 . 2}$ & 88.5 & 92.2 \\
Capsule-A & 79.5 & 92.6 & 90.2 & 91.4 \\
AT-CapsNet & 82.4 & 92.2 & 91.0 & 91.6 \\
Syntax-AT-Capsule(ours) & $\mathbf{8 2 . 9}$ & 93.9 & $\mathbf{9 2 . 6}$ & $\mathbf{9 3 . 2}$ \\
\hline Data set & & & & \\
\hline TextCNN & 89.5 & $\mathbf{9 8 . 2}$ & 90.6 & 94.2 \\
Capsule-A & 88.7 & 94.0 & 92.0 & 93.0 \\
AT-CapsNet & 89.9 & 96.8 & 93.2 & 95.0 \\
Syntax-AT-Capsule(ours) & $\mathbf{9 0 . 6}$ & 97.3 & $\mathbf{9 5 . 3}$ & $\mathbf{9 6 . 3}$ \\
\hline
\end{tabular}




\section{Table 3 (on next page)}

The experimental result of multi label classification. Our model is compared with the benchmark model on two multi-label datasets. The evaluation index is ER, Precision, Recall and F1. 


\begin{tabular}{ccccc}
\hline Evaluation index & ER(\%) & Precision $(\%)$ & Recall $(\%)$ & F1(\%) \\
\hline Data set & \multicolumn{4}{c}{ Reuters-Full } \\
\hline TextCNN & 85.0 & $\mathbf{9 7 . 0}$ & 86.5 & 91.4 \\
Capsule-A & 84.5 & 92.5 & 90.2 & 91.9 \\
AT-CapsNet & 86.6 & 94.9 & 90.6 & 92.7 \\
Syntax-AT-CapsNet(ours) & $\mathbf{8 8 . 1}$ & 95.0 & $\mathbf{9 1 . 8}$ & $\mathbf{9 3 . 4}$ \\
\hline Data set & \multicolumn{5}{c}{ Reuters-Multi } \\
\hline TextCNN & 28.7 & $\mathbf{9 6 . 0}$ & 59.1 & 73.2 \\
Capsule-A & 44.3 & 86.3 & 75.6 & 80.6 \\
AT-CapsNet & 64.3 & 88.5 & 84.6 & 86.5 \\
Syntax-AT-CapsNet(ours) & $\mathbf{7 0 . 3}$ & 95.2 & $\mathbf{8 4 . 9}$ & $\mathbf{8 9 . 7}$ \\
\hline
\end{tabular}

1 


\section{Table 4 (on next page)}

The experimental result of Syntax module verification. Our syntactic module was added to the benchmark model and experimented on Reuters-Full datasets. The evaluation index is ER, Precision, Recall and F1. In each control group, the first model has no syn 


\begin{tabular}{ccccc}
\hline Data set & \multicolumn{4}{c}{ Reuters-Full } \\
\hline Evaluation index & ER(\%) & Precision(\%) & Recall(\%) & F1(\%) \\
\hline TextCNN & 85.0 & 97.0 & 86.5 & 91.4 \\
Syntax-CNN & $\mathbf{8 5 . 9}$ & $\mathbf{9 7 . 1}$ & $\mathbf{8 7 . 7}$ & $\mathbf{9 2 . 1}$ \\
\hline Capsule-A & 84.5 & 92.5 & 90.2 & 91.9 \\
Syntax-CapsNet & $\mathbf{8 5 . 4}$ & $\mathbf{9 3 . 1}$ & $\mathbf{9 1 . 7}$ & $\mathbf{9 2 . 4}$ \\
\hline AT-CapsNet & 86.6 & 94.9 & 90.6 & 92.7 \\
Syntax-AT-CapsNet(ours) & $\mathbf{8 8 . 1}$ & $\mathbf{9 5 . 0}$ & $\mathbf{9 1 . 8}$ & $\mathbf{9 3 . 4}$ \\
\hline
\end{tabular}




\section{Table 5 (on next page)}

The experimental result of control single module ablation. Remove one module from our model to verify the effect of the module. There is no corresponding module for the first model in each control group. 


\begin{tabular}{ccccc}
\hline Data set & \multicolumn{5}{c}{ Reuters-Full } \\
\hline Evaluation index & ER(\%) & Precision(\%) & Recall(\%) & F1(\%) \\
\hline \multicolumn{5}{c}{ With/without syntax module } \\
AT-CapsNet & 86.6 & 94.9 & 90.6 & 92.7 \\
Syntax-AT-CapsNet(ours) & $\mathbf{8 8 . 1}$ & $\mathbf{9 5 . 0}$ & $\mathbf{9 1 . 8}$ & $\mathbf{9 3 . 4}$ \\
Decrease value & 1.5 & 0.1 & 1.2 & 0.7 \\
\hline Syntax-CapsNet & With/without attention module & \\
\hline Syntax-AT-CapsNet(ours) & 85.4 & 93.1 & 91.7 & 92.4 \\
Decrease value & $\mathbf{8 8 . 1}$ & $\mathbf{9 5 . 0}$ & $\mathbf{9 1 . 8}$ & $\mathbf{9 3 . 4}$ \\
\hline Syntax-AT-CNN & 2.7 & 1.9 & 0.1 & 1 \\
\hline Syntax-AT-CapsNet(ours) & $\mathbf{8 8 . 1}$ & $\mathbf{9 5 . 0}$ & 91.0 & 92.5 \\
Decrease value & 2.9 & 1 & $\mathbf{9 1 . 8}$ & $\mathbf{9 3 . 4}$ \\
\hline
\end{tabular}

1 


\section{Table 6(on next page)}

The experimental result of control two module ablation. Remove two module from our model to verify the effect of the module. There is no corresponding module for the first model in each control group. 


\begin{tabular}{|c|c|c|c|c|}
\hline Data set & \multicolumn{4}{|c|}{ Reuters-Full } \\
\hline Evaluation index & $\mathrm{ER}(\%)$ & Precision $(\%)$ & Recall(\%) & $\mathrm{F} 1(\%)$ \\
\hline \multicolumn{5}{|c|}{ With/without syntax + attention module } \\
\hline CapsNet & 84.5 & 92.5 & 90.2 & 91.9 \\
\hline Syntax-AT-CapsNet(ours) & 88.1 & 95.0 & 91.8 & 93.4 \\
\hline Decrease value & 3.6 & 2.5 & 1.6 & 1.5 \\
\hline \multicolumn{5}{|c|}{ With/without syntax + capsule network module } \\
\hline $\mathrm{AT}-\mathrm{CNN}$ & 84.0 & 94.3 & 87.5 & 90.8 \\
\hline Syntax-AT-CapsNet(ours) & 88.1 & 95.0 & 91.8 & 93.4 \\
\hline Decrease value & 4.1 & 0.7 & 4.3 & 2.6 \\
\hline \multicolumn{5}{|c|}{ With/Without Attention + Capsule Network Module } \\
\hline Syntax-CNN & 85.9 & 97.1 & 87.7 & 92.1 \\
\hline Syntax-AT-CapsNet(ours) & 88.1 & 95.0 & 91.8 & 93.4 \\
\hline Decrease value & 2.2 & -2.1 & 4.1 & 1.3 \\
\hline
\end{tabular}

1 